\title{
Immunohistochemical Localization of Endothelial Nitric Oxide Synthase in Endometrial Tissues of Women With Uterine Myomas
}

\author{
Marefat Ghaffari Novin $^{1,2}$, Ashraf Moini ${ }^{3}$, Sogol Niknezhad ${ }^{1}$, Tohid Najafi ${ }^{1,4^{*}}$, Mahsa Ghaffari Novin ${ }^{5}$
}

\begin{abstract}
Objectives: Nitric oxide (NO) is a free radical that plays important roles in variety of physiological aspects of the female reproductive system. Pathophysiological findings revealed a potential role of the endothelial isoform of nitric oxide synthase (eNOS) enzyme in female reproductive disorders specifically in the endometrium. This study investigates the expression of eNOS in the endometrial tissue to study the potential role of this enzyme and its NO production in infertility of women with uterine myomas.

Materials and Methods: A total of 20 endometrial tissues were obtained, 10 infertile women with uterine fibroids and 10 from normal and fertile women, 7 to 9 days after LH surge. Following fixation with paraformaldhyde, frozen sections of samples were prepared for semi-quantitative immunohistochemical evaluation using monoclonal anti-human eNOS antibody. Hematoxylin and eosin staining was used for histological dating of the samples

Results: Localization of eNOS was seen in glandular and luminal epithelium, vascular endothelium and stroma in both fertile women and infertile women with uterine fibroids. Despite the differences in immunoreactivity of luminal epithelium, vascular endothelium and stroma in both groups, higher levels of eNOS in glandular epithelium was statistically significant in women with uterine fibroids compared to the control group.

Conclusions: The findings suggest that over-expression of eNOS in glandular epithelium may affect the preparation stage of endometrium for fertility in women with uterine myomas.

Keywords: Nitric oxide, Endothelial nitric oxide synthase, Endometrium, Uterine myomas, Fibroids
\end{abstract}

\section{Introduction}

Uterine myomas or fibroids are known as the most prevalent benign solid tumors of the female reproductive system. They are commonly found in the myometrial layer of the uterus; however their presence in other different locations both inside and outside the uterus has been reported. Although myomas are very prevalent and are diagnosed in $25 \%-50 \%$ of females, the exact pathogenesis has not been determined; however, it has been reported that these tumors are derived from myoma cells by the intervention of specific hormones (1). Uterine myomas can diversely affect normal pathophysiology of reproductive system and cause pain, infertility, pregnancy loss, menorrhagia and many other complications through different mechanisms. Moreover, they could reduce the efficacy of assisted reproductive technologies and the implantation rate (2). Studies have proven that intramural and submucosal fibroids could lower the rate of fertility (3). Since 1980, surgical myomectomy is routinely used as a common treatment procedure for patients with uterine myomas; this technique has been approved for its outstanding advantages in medical, social and economic terms by decreasing morbidity (4). However, in many cases, due to their asymptomatic nature, uterine myomas do not necessarily need to be removed.

Nitric oxide (NO) is a free radical that plays diverse physiological roles in human systems. Nitric oxide synthase (NOS) is the name given to a group of enzymes which produce NO from L-arginine amino acid. In the human endometrial tissue, all three isoforms of NOS (iNOS, eNOS and nNOS) are expressed; However the endothelial isoform (eNOS) is the predominant one(5). To date, we do know eNOS acts as a regulator of uterine quiescence during the gestational period and studies have demonstrated its role in signal transduction pathways (6) as well as relaxation of myometrial smooth muscles $(7,8)$. Even though the release of eNOS is a critical factor to maintain pregnancy, over-expression of this enzyme has been shown to induce cellular apoptosis (9) and/or impair endometrial and myometrial function by nitrosylation of key endometrial proteins $(10,11)$ as well as subsequent effects on myometrial relaxation. The same conditions also have been reported in the state of endometriosis (12-14), adenomyosis (15), unexplained infertility, and 
recurrent miscarriage (16). It has been demonstrated that estrogen stimulates the proliferation of the uterine fibroids by direct activation of the uterine fibroblast cells (17). Additionally, studied have shown that estrogen and progesterone are the major regulators of expression of eNOS in the human endometrium (18-20). Thus, in regard to estrogen-dependent pathology of the uterine fibroids, it seems that NO levels may play a considerable role in the formation of uterine fibroids. These alterations in the enzyme expression, can lead to formation of oxidative stress, which has been shown to cause reproductive disorders (21,22). Accordingly, we hypothesize that overexpression of endometrial eNOS could occur in patients with uterine myomas and potentially affect their fertility. The role of NO in uterine myomas has been studied by a few studies; however all of those have focused on the fibroid tissues per se as well as myometrium and thus the potential resultant endometrial alteration of the enzyme remained unclear $(23,24)$

As the main expressed isoform of NOS in musculature and endothelium of myometrial vessels, eNOS can be potentially involved in the pathophysiology of endometrial layer. Endothelial nitric oxide synthase (eNOS) plays an imperative role in the maintenance of uterine quiescence and NO produced by the action of this enzyme can be involved in diverse physiological aspects of gestation. Therefore, we sought to clarify whether expression of eNOS is altered in patients with uterine fibroids and whether (if any) it can influence the process of implantation.

\section{Materials and Methods \\ Study Design}

The study group was asked to sign a consent form for their participation. In this randomized study, women in the control group $(n=10)$ with no history of urogenital conditions including uterine myomas and endometriosis experienced at least one natural labor and had normal menstrual cycles and had not received any hormonal medications for at least 4 months before sample collection. This women voluntarily participated in this study upon their attendance for regular process of gynecological health check and mean age of those women was 33.9 years (range, 29-39 years). Uterine fibroids in myometrium of experiment group $(n=10)$ had been diagnosed by ultrasonography and hysterosalpingography (6 intramural, 4 submucosal).Within this group, patients who had reasons of infertility other than uterine myomas such as endometriosis, adenomyosis, abnormal endocrine findings (TSH, cycle-day-3 FSH and estradiol concentrations, prolactin and progesterone concentrations) were excluded from the study. The study group was selected from among those women with uterine myomas, which resumed their fertility after myomectomy by report of at least one successful pregnancy and regardless of miscarriage to ensure that their infertility was truly myoma-based or at least in very close relationship with fibroid formation.

Sample Collection and Preparation of Sections

Biopsy samples of endometrium were obtained by using a Pipelle curette 7 to 9 days after the urinary $\mathrm{LH}$ surge at the sites close to the myomectomy following Cuntz procedure (25). Since the plan of this study was to analyze the eNOS change in patients with uterine fibroids from the implantation point of view, all of the phases of the post-ovulatory samples were determined by ultrasound to ensure about the correct timing of sample collection. At first obtained samples were treated with $4 \%$ paraformaldhyde tissue fixative for maximum 24 hours followed by rinsing in $70 \%$ ethanol and later stored at $-20^{\circ} \mathrm{C}$ until processing. Histological dating was done by using the criteria of Noyes et al (26). Each biopsy specimen was later cut into $6 \mu \mathrm{m}$ sections by using a Reichert-Jung Cryocut 1800 (Cambridge Instruments GmbH, Nussloch, Germany) and place on poly l-lysin coated slides and store in $-70^{\circ} \mathrm{C}$ until immunostaining.

\section{Quantitative Immunohistochemistry}

For microscopic detection of the intensity and distribution of eNOS immunostaining, a monoclonal mouse antihuman antibody (6H2, cat. no. 91205; ABcam Co, USA) was applied. Rinsing with buffer ( $0.1 \mathrm{M}$ of phosphate-buffered saline with a $\mathrm{pH}$ of 7.4 ) followed by incubation in $0.3 \%$ $\mathrm{H} 2 \mathrm{O} 2$ in methanol for 20 minutes to quench endogenous peroxidases. Rinsing repeated with $0.05 \%$ bovine serum albumin in phosphate-buffered saline and retrieval of antigens was done by adding tripsin. Afterwards, the slides were exposed to $1.5 \%$ normal horse serum (Sigma Chemical Co., St. Louis, MO) in humidified chambers for 30 minutes at room temperature. The primary antibody against eNOS (1:100) was placed on slides and incubated at $37^{\circ} \mathrm{C}$ for $1 \mathrm{~h}$. After binding of the primary antibody, the sections were incubated with the secondary antibody, a rabbit antimouse IgG (ABcam Co. USA) diluted in PBS. Incubation with the secondary antibody was performed for $1 \mathrm{~h}$ at room temperature. 3,3'-diaminobenzidine in $\mathrm{H} 2 \mathrm{O} 2$ (Vector Laboratories, Inc.) was added to the sections for 10 minutes. Thereafter, the sections were counterstained with hematoxylin and mounted. For negative controls, using same method, phosphate-buffered saline replaced the primary antibody.

Immunohistochemical staining was evaluated quantitatively by 2 independent examiners by using Image-Pro Plus software (Figure 1) using previously described methods (27) on figures of Zeiss light microscope (Axioskop, Zeiss, Göttingen, Germany). Four different areas of the sections were analyzed at varying magnifications and the mean was used for statistical analysis. To avoid errors due to uneven staining, 2 sections of each endometrial biopsy specimen including both epithelial and stromal cells were evaluated. We used human placental sections as positive controls which 


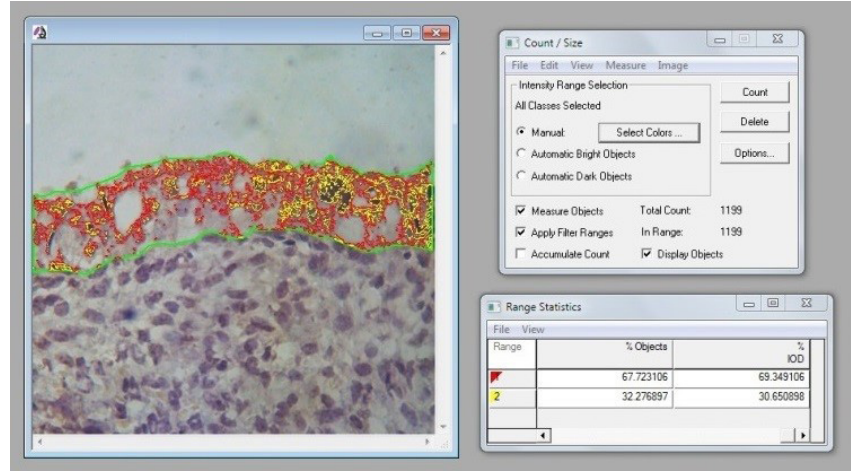

Figure 1. Image Pro Plus software used to determine staining intensity of sections. immunohistochemical staining reported as intensity of optic density (IOD\%).

showed normal immunostaining (28).

\section{Statistical Analysis}

Differences in immunohistochemical staining between control and experimental groups as intensity of optic density (IOD\%) were analyzed by using the exact ManWhitney $U$ test of SPSS software. Results have been reported as mean standard deviation and $P \leq 0.05$ was considered statistically significant.

\section{Results}

eNOS Immunolocalization in Endometrium

The demographics of the 2 groups of study are shown in Table 1. None of the subjects had any chronic medical illness and laparoscopic evaluation and hormonal tests showed normal anatomy and endocrinology of all subjects. There was also a small difference in age and body mass index between two groups which were not statistically significant. eNOS was localized in vascular endothelium, glandular epithelium, luminal epithelium and the stroma (Figure 2). Expression of eNOS in endometrium of infertile women changed in vascular endothelium $(P=0.65)$, glandular epithelium $(P=0.045)$, luminal epithelium $(P=0.44)$, and stroma $(P=0.60)$.

Accordingly, despite the differences between expression of eNOS in luminal epithelium, stroma and vascular endothelium only the difference between two groups in glandular epithelium was statistically considerable $(P=0.045$; Figure 3$)$.

\section{Discussion}

Known as the most common benign uterine tumor in uterus of women of reproductive age, uterine myomas or fibroids are hormone dependent (especially estrogen and progesterone) tumors that manifest largely in the myometrial layer of uterus and in most cases have to be extruded surgically due to their approved negative effects on reproductive health based upon the location and physical characteristics (29).

We detected eNOS in blood vessels as well as glandular and luminal epithelial cells and endometrial stroma. These findings are in accordance with previous studies by Najafi et al (16) and Tefler et al (30). It has also been reported previously that at the time of expected implantation, the endothelial isoform of NOS is expressed dominantly in the human endometrium (18). By continues release of NO into the lumen, processes such as menstruation and implantation are facilitated through prostaglandin synthesis and modulation of anchoring proteins. On the other side, eNOS-derived NO, may function as an inhibitor of endometrial platelet aggregation by activating the soluble guanylyl cyclase formation or through cyclooxygenase catalysis (31).

Our previous studies showed that glandular epithelium releases most eNOS from the endometrial tissue in normal and infertile women with unknown etiologies $(16,32)$. This finding was confirmed in the present work. Although this finding is partially in contrast with that of Taguchi (33), reporting that vascular endothelium is the major part of eNOS expression, we see a small increase in the endothelial expression of the enzyme in the vessels. This increased local NO expression could cause activation of cyclooxygenase- 2 and subsequently elevating the prostaglandins levels, such as prostaglandin E2 (34). On the other hand, the vascular expression of eNOS in our study, approves the findings by Gokdeniz et al,

Table 1. Characteristics of study group $(n=10)$ and control group $(n=10)$

\begin{tabular}{|c|c|c|c|c|c|c|c|}
\hline & Age (y) & $\begin{array}{c}\text { BMI } \\
\left(\mathrm{kg} / \mathrm{m}^{2}\right)\end{array}$ & $\begin{array}{l}\text { Live Birth } \\
\qquad(\mathrm{n})^{*}\end{array}$ & $\begin{array}{l}\text { Infertility } \\
\text { (y) } *\end{array}$ & $\begin{array}{l}\text { Cigarette } \\
\text { Smoke }\end{array}$ & $\begin{array}{c}\text { No. of Pregnancy } \\
\text { Prior to Myomectomy }\end{array}$ & $\begin{array}{l}\text { Number of Pregnancy } \\
\text { Following Myomectomy }\end{array}$ \\
\hline Control & $\begin{array}{c}33.9 \\
S D=1.8\end{array}$ & $\begin{array}{c}24.59 \\
S D=2.3\end{array}$ & $\begin{array}{c}1.60 \\
\mathrm{SD}=2.1\end{array}$ & 0 & 0 & $\begin{array}{c}2.8 \\
\mathrm{SD}=1.8\end{array}$ & N/A \\
\hline Uterine myomas & $\begin{array}{c}32.3 \\
S D=1.6\end{array}$ & $\begin{array}{l}24.05 \\
S D=2.00\end{array}$ & 0.00 & $\begin{array}{c}3.10 \\
S D=2.0\end{array}$ & 0 & 0 & $\begin{array}{c}1.3 \\
S D=2.00\end{array}$ \\
\hline
\end{tabular}

Abbreviation: $\mathrm{SE}=$ standard error. 


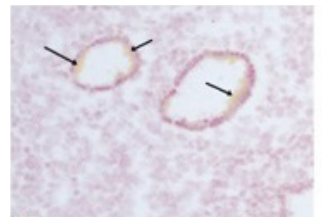

A
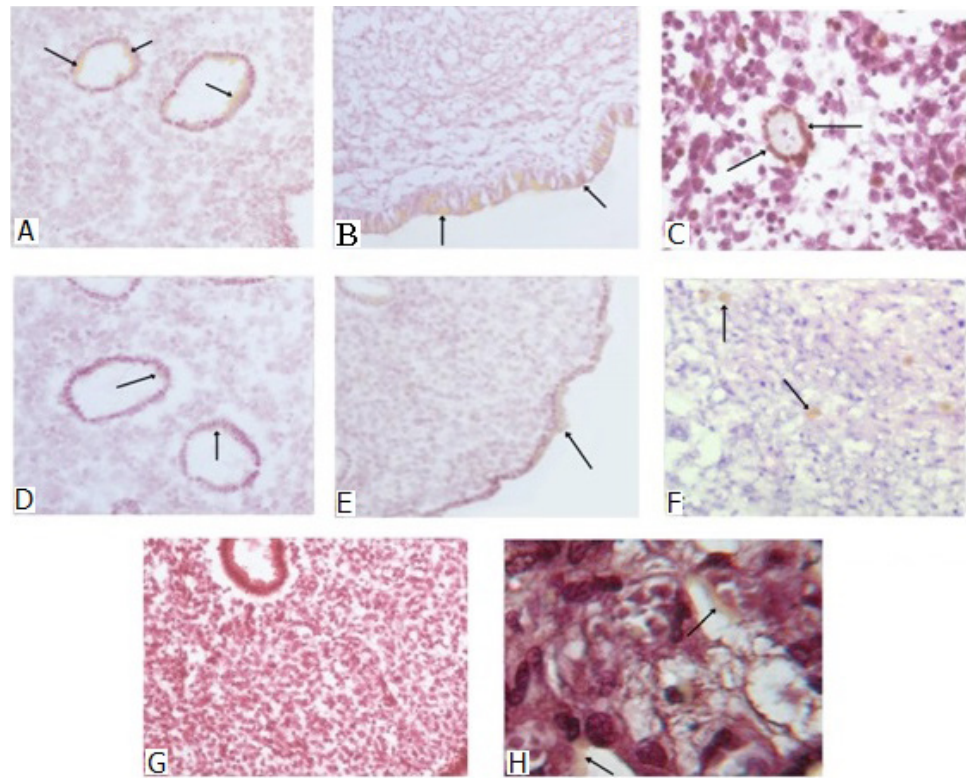

Figure 2. Immunostaining of eNOS in different endometrial compartments of the study groups. Glandular epithelium (A) and luminal epithelium (B) and vascular endothelium (C) of a myoma. Glandular epithelium (D), luminal epithelium (E) and stroma $(F)$ of a control subject. Negative (G) and positive $(\mathrm{H})$ controls in a human endometrium and placental sections respectively.

Arrows indicate areas with positive eNOS immunostaining. Magnification for epithelium is $10(A, B, D, E, G)$ and for endothelium and stroma is 40 $(\mathrm{C}, \mathrm{F}, \mathrm{H})$

nevertheless their study mainly focuses on the muscular characteristics of myoma tissues (35)

Although this study shows a non-significant increase in the expression of enzyme in vascular endothelium, this finding is not in contrast with results of Oh et al study (36) which found significant over-expression of enzyme in vasculature of the endothelium. Vascular over-expression of eNOS in case of uterine myoma may affect the biological integrity of endometrium. It has been shown that unpaired electron of extremely reactive NO molecule

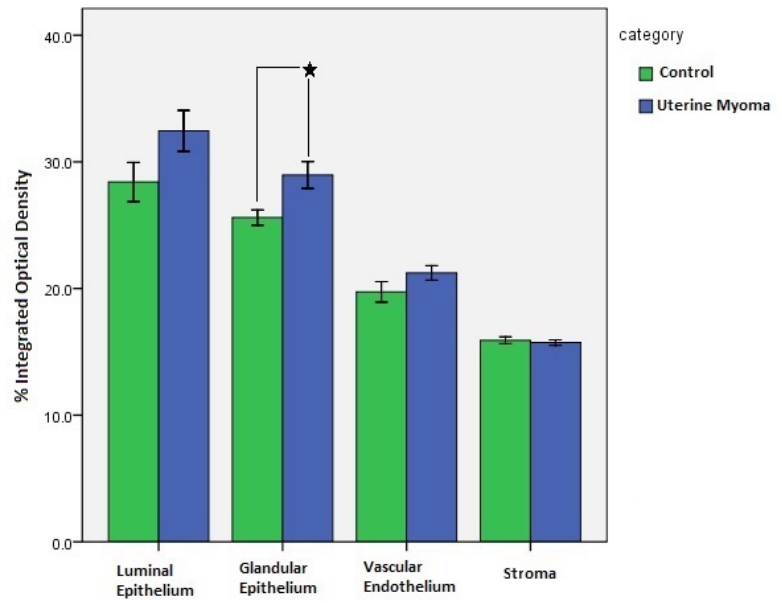

Figure 3. eNOS Protein Quantification by Image Analysis In 4 Compartments of the Endometrium in Control, and Uterine Myoma Groups. The expression of eNOS in Jlandular epithelium has been significantly increased. On the other hand, in spite of relatively large differences in luminal epithelium, the higher standard errors in this group indicate the insignificancy of the difference by Mann Whitney $U$ test. ${ }^{*} P<0.05$. Error Bars $=+/-1 \mathrm{SE}$. in higher amounts, are potentially able to damage the structure of protein, carbohydrates, nucleotides and lipids (21). Accordingly for uterine fibroids, we can assume a mechanism similar to that which occurs in inflammatory conditions such as endometrial adhesions by inducing oxidative stress. But due to different reported amount of the enzyme, it seems that the expression of eNOS in endometrial vessels varies by location and/or menstrual date.

Apart from the vessels wall, the presence of eNOS in luminal epithelium and stromal cells of women with uterine fibroids may be the indicative of the role of NO in the control of endometrial function. But based on the findings of this study we cannot claim that if pregnancy occurs, even for a short duration of time, this role may be interrupted. It seems that like endometrial tissues of women with unexplained infertility and recurrent pregnancy loss, the cyclo-oxygenase pathway, $\mathrm{NO}$ and cytokines which interact to regulate uterine function (34) have also been undisrupted in women with uterine fibroids.

Although NO in its physiological release, acts specifically on the myometrial tissue to cause muscle relaxation at the end stage of labor, changes in the endometrial expression of eNOS, as the major isoform of NOS in the females' reproductive tract, can demonstrate the activity log of this free radical in the lumen of uterus in specific malignancy conditions.

From the window of implantation, it has been strongly reported that uterine fibroids could affect various stages of fertility especially early implantation which can result in infertility and miscarriage (3). Several studies 
have demonstrated that increase in the expression of prostaglandins $\mathrm{E}$ and $\mathrm{F}$ and $\mathrm{NO}$ occurs as complementary supplements of implantation $(37,38)$ and from this point of view, we can suggest that due to infertility outcomes of uterine fibroids, the increase in glandular epithelium may act as a compensatory mechanism to overcome unwanted results of uterine myomas on implantation and embryo-endometrial adhesions. But further studies are needed to investigate if suppression of eNOS activity by nitro-L-arginine methylester (L-NAME), a common NOS blocker, can cause the changes in the implantation and fertility of patient with uterine myomas. Taking the implantation failure secondary to endometrial factors in unexplained infertility (39) and recurrent miscarriage (40) into consideration, the present study suggests that an irregular endometrial expression of eNOS in women with uterine fibroids may result in implantation failure through the similar mechanisms.

The expression levels of eNOS in vascular endothelium were not significantly different compared to the control group, however, the low expression of the enzyme, as seen in these endometrial samples, could be considered as a potential factor leading to infertility and miscarriage subsequent to myoma formation. This suggests that aberrant production of eNOS in non-endothelial areas (especially glandular epithelium) may play a more remarkable role in the pathophysiology of uterine fibroids compared to endothelial areas. Although we used specialized software to detect fold changes in enzyme expression, it can be more useful to confirm this data with quantitative protein analysis such as blotting techniques, which may be considered as a limitation of our study due to difficulties in collection of endometrial samples.

In summary, the present data by demonstrating an overexpression of eNOS in the endometrium of fibroid uteri, suggests that excess expression of eNOS (and subsequent excess generation of NO in the endometrium) in patients with uterine myomas may cause nitrosative stress which in turn could result in infertility or miscarriage or even preterm birth; However we do know that expression of eNOS to some extent is essential for implantation and fertilization. It seems that this disordered expression of eNOS is similar in inflammation pattern to other gynecological conditions such as endometriosis and adenomyosis and can be considered as a potential component resulting in infertility and/or miscarriage. However, larger and comprehensive studies with concentration on NOS protein and gene expressions are needed to explore exact mechanisms of $\mathrm{NO}$ action in the endometrial tissue in these women.

\section{Conflict of Interests}

Authors declare that they have no conflict of interests.

\section{Ethical Issues}

The protocol for this randomized study was approved by the Ethics and Human Subjects Committee at Shahid Beheshti University of Medical Sciences (91st Session, 2011).

\section{Financial Support}

This research was supported by Infertility and reproductive health researches Center at Shahid Beheshti University of Medical Sciences.

\section{Acknowledgment}

The authors sincerely thank Dr. Mojgan Bandehpour and Mrs.Gelareh Rahimi for their advice regarding sample preparations and statistical tests.

\section{References}

1. Yoshino O, Hayashi T, Osuga Y, Orisaka M, Asada H, Okuda $\mathrm{S}$, et al. Decreased pregnancy rate is linked to abnormal uterine peristalsis caused by intramural fibroids. Hum Reprod 2010; 25: 2475-9. doi: 10.1093/humrep/deq222

2. Stovall DW, Parrish SB, Van Voorhis BJ, Hahn SJ, Sparks AE, Syrop CH. Uterine leiomyomas reduce the efficacy of assisted reproduction cycles: results of a matched follow-up study. Hum Reprod 1998; 13: 192-7.

3. Pritts EA, Parker WH, Olive DL. Fibroids and infertility: an updated systematic review of the evidence. Fertil Steril 2009; 91: 1215-23. doi: 10.1016/j.fertnstert.2008.01.051

4. Semm K, Mettler L. Technical progress in pelvic surgery via operative laparoscopy. Am J Obstet Gynecol 1980; 138: 121-7.

5. Khorram O, Garthwaite M, Magness RR. Endometrial and myometrial expression of nitric oxide synthase isoforms in pre- and postmenopausal women. J Clin Endocrinol Metab 1999; 84: 2226-32. doi: 10.1210/jcem.84.6.5759

6. Thomas DD, Ridnour LA, Isenberg JS, Flores-Santana W, Switzer CH, Donzelli S, et al. The chemical biology of nitric oxide: implications in cellular signaling. Free Radic Biol Med 2008; 45: 18-31. doi: 10.1016/j.freeradbiomed.2008.03.020

7. Buxton IL. Regulation of uterine function: a biochemical conundrum in the regulation of smooth muscle relaxation. Mol Pharmacol 2004; 65: 1051-9. doi: 10.1124/ mol.65.5.1051

8. Norman JE, Ward LM, Martin W, Cameron AD, McGrath JC, Greer IA, et al. Effects of cGMP and the nitric oxide donors glyceryl trinitrate and sodium nitroprusside on contractions in vitro of isolated myometrial tissue from pregnant women. J Reprod Fertil 1997; 110: 249-54.

9. Wang Y, Chen C, Loake GJ, Chu C. Nitric oxide: promoter or suppressor of programmed cell death? Protein Cell 2010; 1: 133-42. doi: 10.1007/s13238-010-0018-x

10. Gu Z, Nakamura T, Lipton SA. Redox reactions induced by nitrosative stress mediate protein misfolding and mitochondrial dysfunction in neurodegenerative diseases. Mol Neurobiol 2010; 41: 55-72. doi: 10.1007/s12035-0108113-9

11. Weiner D, Khankin EV, Levy Y, Reznick AZ. Effects of cigarette smoke borne reactive nitrogen species on salivary alpha-amylase activity and protein modifications. J Physiol Pharmacol 2009; 60 Suppl 5: 127-32.

12. Dong $M$, Shi $Y$, Cheng $Q$, Hao M. Increased nitric oxide in 
peritoneal fluid from women with idiopathic infertility and endometriosis. J Reprod Med 2001; 46: 887-91.

13. Khorram O, Lessey BA. Alterations in expression of endometrial endothelial nitric oxide synthase and alpha(v) beta(3) integrin in women with endometriosis. Fertil Steril 2002; 78: 860-4.

14. Wu MY, Chao KH, Yang JH, Lee TH, Yang YS, Ho HN. Nitric oxide synthesis is increased in the endometrial tissue of women with endometriosis. Hum Reprod 2003; 18: 2668-71.

15. Ota H, Igarashi S, Hatazawa J, Tanaka T. Endothelial nitric oxide synthase in the endometrium during the menstrual cycle in patients with endometriosis and adenomyosis. Fertil Steril 1998; 69: 303-8.

16. Najafi T, Novin M, Ghazi R, Khorram O. Altered endometrial expression of endothelial nitric oxide synthase in women with unexplained recurrent miscarriage and infertility. Reprod Biomed Online 2012; 25: 408-14. doi: 10.1016/j.rbmo.2012.07.004

17. Luo N, Guan Q, Zheng L, Qu X, Dai H, Cheng Z. Estrogenmediated activation of fibroblasts and its effects on the fibroid cell proliferation. Transl Res 2014; 163: 232-41. doi: 10.1016/j.trsl.2013.11.008

18. Han G, Magee T, Khorram O. Regulation of nitric oxide synthase isoforms by estrogen in the human endometrium. Fertil Steril 2005; 84 Suppl 2: 1220-7. doi: 10.1016/j. fertnstert.2005.06.016

19. Khorram O, Han G. Influence of progesterone on endometrial nitric oxide synthase expression. Fertil Steril 2009; 91: 2157-62. doi: 10.1016/j.fertnstert.2008.05.019

20. Zervou S, Klentzeris LD, Old RW. Nitric oxide synthase expression and steroid regulation in the uterus of women with menorrhagia. Mol Hum Reprod 1999; 5: 1048-54.

21. Agarwal A, Gupta S, Sekhon L, Shah R. Redox considerations in female reproductive function and assisted reproduction: from molecular mechanisms to health implications. Antioxid Redox Signal 2008; 10: 1375-403. doi: 10.1089/ ars.2007.1964

22. Fletcher NM, Saed MG, Abuanzeh S, Abu-Soud HM, AlHendy A, Diamond MP, et al. Nicotinamide Adenine Dinucleotide Phosphate Oxidase Is Differentially Regulated in Normal Myometrium Versus Leiomyoma. Reprod Sci 2014. doi: $10.1177 / 1933719114522552$

23. Plewka A, Madej P, Plewka D, Kowalczyk A, Miskiewicz A, Wittek P, et al. Immunohistochemical localization of selected pro-inflammatory factors in uterine myomas and myometrium in women of various ages. Folia Histochem Cytobiol 2013; 51: 73-83. doi: 10.5603/FHC.2013.0011

24. Favini R, Aldieri E, Revelli A, Bosia A, Massobrio M, Ghigo D. Nitric oxide synthesis in human nonpregnant myometrium and uterine myomas. Fertil Steril 2003; 79 Suppl 1: 749-53.

25. Kuntz C. Practice tips. Endometrial biopsy. Can Fam
Physician 2007; 53: 43-4.

26. Noyes RW, Hertig AT, Rock J. Dating the endometrial biopsy. Am J Obstet Gynecol 1975; 122: 262-3.

27. Khorram O, Khorram N, Momeni M, Han G, Halem J, Desai $\mathrm{M}$, et al. Maternal undernutrition inhibits angiogenesis in the offspring: a potential mechanism of programmed hypertension. Am J Physiol Regul Integr Comp Physiol 2007; 293: R745-53. doi: 10.1152/ajpregu.00131.2007

28. Bhuiyan MB, Murad F, Fant ME. The placental cholinergic system: localization to the cytotrophoblast and modulation of nitric oxide. Cell Commun Signal 2006; 4: 4. doi: 10.1186/1478-811X-4-4

29. Lumsden MA, Hamoodi I, Gupta J, Hickey M. Fibroids: diagnosis and management. BMJ 2015; 351: h4887. doi: 10.1136/bmj.h4887

30. Telfer JF, Irvine GA, Kohnen G, Campbell S, Cameron IT. Expression of endothelial and inducible nitric oxide synthase in non-pregnant and decidualized human endometrium. Mol Hum Reprod 1997; 3: 69-75.

31. Chwalisz K, Garfield RE. Role of nitric oxide in implantation and menstruation. Hum Reprod 2000; 15 Suppl 3: 96-111.

32. Najafi T NM, Pakravesh J, Foghi K, Fadayi F, Rahimi G. Immunohistochemical localization of endothelial nitric oxide synthase in endometrial tissue of women with unexplained infertility. Iran J Reprod Med 2012; 10: 121-6.

33. Taguchi M, Alfer J, Chwalisz K, Beier HM, Classen-Linke I. Endothelial nitric oxide synthase is differently expressed in human endometrial vessels during the menstrual cycle. Mol Hum Reprod 2000; 6: 185-90.

34. Dong YL, Yallampalli C. Interaction between nitric oxide and prostaglandin E2 pathways in pregnant rat uteri. Am J Physiol 1996; 270: E471-6.

35. Gokdeniz R, Mizrak B, Ozen S, Bazoglu N. Endothelial nitric oxide synthase expression in leiomyoma and parental myometrium. Gynecol Obstet Invest 2000; 49: 132-6. doi: 10231

36. Oh NJ, Ryu KY, Jung CN, Yi SY, Kim SR. Expression of endothelial nitric oxide synthase in the uterus of patients with leiomyoma or adenomyosis. J Obstet Gynaecol Res 2013; 39: 536-42. doi: 10.1111/j.1447-0756.2012.01980.x

37. Khorram O. Nitric oxide and its role in blastocyst implantation. Rev Endocr Metab Disord 2002; 3: 145-9.

38. Kennedy TG, Gillio-Meina C, Phang SH. Prostaglandins and the initiation of blastocyst implantation and decidualization. Reproduction 2007; 134: 635-43. doi: 10.1530/REP-07-0328

39. Koot YE, Boomsma CM, Eijkemans MJ, Lentjes EG, Macklon NS. Recurrent pre-clinical pregnancy loss is unlikely to be a 'cause' of unexplained infertility. Hum Reprod 2011; 26: 2636-41. doi: 10.1093/humrep/der217

40. Li TC, Tuckerman EM, Laird SM. Endometrial factors in recurrent miscarriage. Hum Reprod Update 2002; 8: 43-52.

(C) 2018 The Author (s); This is an open-access article distributed under the terms of the Creative Commons Attribution License (http://creativecommons.org/licenses/by/4.0), which permits unrestricted use, distribution, and reproduction in any medium, provided the original work is properly cited. 